\title{
SOBRE LA EUTANASIA
}

\section{ABOUT EUTHANASIA}

David Caldevilla Domínguez: Profesor del Área de Comunicación en la Universidad Complutense de Madrid, la Universidad Europea de Madrid y Escuela Superior de Estudios de Relaciones Públicas. david.caldevilla@ccinf.ucm.es

\section{CURRÍCULUM VITAE}

Diplomado en Magisterio por la Universidad de Zaragoza (España), licenciado en Comunicación Audiovisual por la Universidad Complutense de Madrid (España). Profesor en la Facultad de Ciencias de la Información de la Universidad Complutense. Secretario General del Fórum Internacional de Comunicación y Relaciones Públicas (España) y autor de varios artículos en revistas universitarias. Director de Operaciones de Imagométrica, s.l. y ex directivo de varias firmas españolas del ámbito de la mercadotecnia.

\section{RESUMEN}

Cuando el director de cine Alejandro Amenábar presentó su film "Mar adentro", la sociedad volvió a hacerse eco del tema de la eutanasia. Surgió de nuevo el debate sobre el aspecto humano de este acto, y si debía complacerse el deseo del afectado u obligarle a vivir contra su voluntad. En este artículo se intentan aclarar algunos de los aspectos capitales sobre qué es y qué no es la eutanasia para que así se forme una opinión pública responsable. 


\title{
PALABRAS CLAVE
}

Eutanasia - Tipos - Opinión Pública

\begin{abstract}
When film director Alejandro Amenabar presented his film "The Sea Inside", the company turned to echo the theme of euthanasia. Came back the debate on the human aspect of this act, and whether to indulge the desire of the affected or force him to live against their will. This article attempts to clear up some of the capital on what is and is not euthanasia so that they form a responsible opinion.
\end{abstract}

\section{KEY WORDS}

Euthanasia - Types - Public Opinion

\section{TEXTO:}

Estos días se viene hablando mucho de lo que está sucediendo en los EE.UU. sobre si la eutanasia es un derecho humano o no. Incluso existe una ramificación hispana en el hospital de Leganés y su programa de sedación a enfermos terminales; pero como dijo Kipling "eso es ya otra historia", y así seguirá por nuestra parte.

Esto viene a colación por el caso de Terri Schiavo, que no ha hecho sino avivar la polémica creada hace años. Terri era una mujer que se hallaba en estado vegetativo desde hacía 15 años y que por propia voluntad expresada a su marido, cuando ésta 
se hallaba en posesión de todas sus facultades, deseaba que no se la mantuviera con vida más allá de lo que fuera natural, es decir, sin ayuda externa.

Ahora se trataba de desconectarla del alimentador artificial que la mantenía entre nosotros para que así pudiera morir por inanición, crueldad que se me antoja más propia de un país tercermundista que de uno desarrollado, pese a que EE.UU., como tal, vive en una perpetua doble moral heredada de sus ancestros puritanos, fuertemente paleotestamentarios, en general defensores de la pena de muerte (irónico cuando menos) y su adecuación a la vida actual. Mucho más humano es procurar una buena muerte o "eu-tanatós" en la lengua de Homero, que es lo que significa la palabra cismática que nos ocupa.

Hace 4 años, entre la Facultad de Ciencias de la Información de la Complutense y otras Universidades nacionales y extranjeras, generamos una investigación exhaustiva mediante el trabajo de numerosos alumnos y profesores. En especial abordamos este fenómeno desde el concepto de imagen que es el que más afecta a nuestros estudios.

Las conclusiones fueron para todos los gustos, como se apostilla de los colores. Comencemos por el principio y juzgue el lector de qué parte del Rubicón se hallan sus legiones.

En puridad, existen diversos tipos de eutanasia, in concreto:

-La eutanasia voluntaria e involuntaria: Involuntaria es la llevada a cabo sin ser solicitada por el enfermo, no la acepta hoy ningún código legal, y mucho menos moral, ético o religioso. Por lo tanto cualquier intento de legalidad parte siempre del \$supuesto de que ha sido voluntariamente aceptada. 
-Piadosa y eugénica: Según sus fines perseguidos, son así llamadas. La piadosa busca privar al enfermo del tormento de los dolores, de una deformación física, una ancianidad penosa, etc., y la eugénica busca la muerte para liberar a la familia o a la sociedad de una vida "sin valor", para purificar la raza, etc. Este tipo de eutanasia sería típico de ideologías totalitarias que consideran al hombre propiedad del estado. Hoy existe legalmente contemplado en muchos países el llamado aborto selectivo que es permitir a la madre abortar antes de que el niño nazca, lo cual es una forma de eutanasia eugénica. Atrás queda el proyecto estatal nazi "fuente de vida" que se presentó ante los teutones y, por ende, ante la comunidad internacional, como una liberación del espíritu que habitaba cuerpos o mentes enfermos sin solución. Se aplicó a enfermos incurables y a retrasados mentales. No confundir con la "solución final".

-La lenitiva y homicida: Depende de la intencionalidad. La lenitiva es la causada indirectamente, al suprimir el dolor, como efecto secundario inevitable (los analgésicos o sedantes se administrarán en cualquier dosificación y por cualquier vía que sea necesaria para mitigar el sufrimiento, aún a riesgo de deprimir la respiración o la tensión arterial, o incluso acortar la vida del paciente) que es el caso de Leganés que no nos ocupa, y la segunda causa directamente la muerte, sin que el fin último sea mitigar al máximo el dolor.

-Pasiva y activa: El término pasiva suele estar mal empleado por los medios de comunicación para masas y a lo único que hace referencia es a la muerte natural, así se suspende el uso de los instrumentos de apoyo de vida o el suministro de medicamentos para que se dé una muerte completamente natural, que no es contraria en nada con la ley natural. Activa se refiere a la muerte que se ocasiona de una manera directa para poner fin a la vida del paciente. 
-Económica: Supone la eliminación de enfermos crónicos o incurables que constituyen una grave carga económica para sus familiares o para la sociedad, ya que ha desaparecido todo tipo de indicio de esperanza en cuanto a su recuperación. Tanto este tipo de eutanasia como la eugénica suponen la muerte obligada del enfermo aún contra su voluntad.

Una vez especificado un vocabulario básico, hagamos un poco de historia sobre la eutanasia y sus mojones itinerarios.

En 1938 se funda la "Eutanasia society of América" cuya meta era legalizar el suicidio con la asistencia de un médico para los enfermos terminales.

A partir de 1945 se revelan las atrocidades nazis (planes de eugenesia racial) que arrojan mucho descrédito sobre la eutanasia.

En 1967 Se presentan los primeros testamentos vitales con el intento de legalizar la eutanasia. Se funda el "Eutanasia Educational Fund" cuyo mayor esfuerzo se encamina a crear una línea de educación pública sobre la eutanasia para verla como una opción aceptable.

En la década de los 70 es fundada la "Society for the right to die" (Sociedad pro el derecho a morir). Estos grupos abogan abiertamente por la eutanasia.

En 1972 se funda el "Eutanasia educational council" (Consejo educativo por la eutanasia).

En 1978 es fundado el "Concern for the dyuing" (Preocupación por morir). 
En 1980 Derek Humphry funda la "Hemlock society" (Sociedad Hemlock) pro eutanasia.

Entre 1985 y 1986 se dan unos casos importantes y notorios que promocionan la eutanasia: Clarie Conroy, Elizabeth Bouvia y Helen Corbett.

En 1986 muere Paul Brophy por inanición, por orden de la Corte Suprema. (Caso similar al de Terri Sciavo).

En 1988 se efectúa una macroencuesta por Roper Poll, cuyos resultados afirman que el $58 \%$ de los estadounidenses están a favor de legalizar morir con la ayuda de un médico.

En 1990 Jack Kevorkian ayuda en un primer suicidio asistido, el de Janet Adkins. La asociación WELS de luteranos pro vida crea un documento de declaración sobre la vida que asesora a los protestantes sobre asuntos ligados al término de la vida. Tras el caso Nancy Cruzan, la Corte Suprema estadounidense declara que los pacientes tienen derecho a rechazar el trato médico. Muere Nancy Cruzan 12 días después de serle retirada la sonda para la alimentación (Caso similar al que nos ocupa de Terri Schiavo). En la nueva encuesta de Roper Poll se lee que el 63\% de los estadounidenses están a favor de legalizar morir con la asistencia médica debida.

En 1991 Derek Humphry escribe La salida final (Final exit) que se mantuvo 18 semanas como uno de los libros más vendidos en EE.UU. según la lista del New York Times. Jack Kevorkian ayuda a otras dos personas a morir. Timothy Quill admite, en un artículo publicado en "The New England Journal of Medicine", que ayudó a un paciente con enfermedad terminal a morir. Un jurado decide no acusarlo de nada. 
En 1992 en el estado de California, los votantes rechazan la proposición 161, claramente pro eutanásica. Jack Kevorkian ayuda a bien morir a otras 5 personas.

En 1993 la nueva encuesta de Harris Poll afirma que el 73\% de los estadounidenses está a favor de legalizar morir con la asistencia médica debida. Holanda es la primera nación en autorizar oficialmente la eutanasia. Muere Christie Busalacchi después de que en el segundo hospital en el que fue internada le retiraran la comida, tras haberle sido negado ese derecho a la familia en el primer hospital. Jack Kevorkian ayuda a otras 12 personas a morir.

En 1994 se plantea un referéndum sobre la Ley de Muerte Digna (Death Dignity Act) para el 8 de noviembre que se pospone al 8 de diciembre. Oregón recusa dicha ley y se opone al referéndum el 7 de diciembre.

En 1995 la WELS luteranos pro vida desarrolla unos documentos sobre la Fuerza duradera de "attorney" por Cuidados de salud, en su versión cristiana.

En 1996 se aprueba en Australia la Ley de derechos para enfermos terminales del Territorio del Norte. Jack Kevorkian declara llevar ya 45 eutanasias.

En 1997 se presentan ante la Corte Suprema estadounidense dos casos de suicidio con asistencia médica. En Australia, en marzo, se anula la Ley de derechos para enfermos terminales del Territorio del Norte. Se aprueba en mayo la Ley de Eutanasia Voluntaria Activa por parte de la Corte Constitucional de Columbia.

Hasta aquí la historia conocida. Las opiniones de los expertos también pueden arrojar luz sobre este asunto. 
Así, P. Martínez Baza y J. Vega Gutiérrez escribieron un trabajo sobre los aspectos legales y deontológicos de la eutanasia. De entre sus ideas destacamos: "Siendo un hecho frecuente la atención sanitaria a los pacientes terminales, generalmente en centros hospitalarios, sorprende la incomodidad del médico y de los enfermeros ante este tipo de pacientes. Ciertamente, cuando la actuación no está orientada a curar la enfermedad o prolongar la vida, el personal sanitario parece encontrarse sin contenido. Se invocan diversas razones para explicar esta actitud frente al enfermo Terminal: ausencia de preparación, reacciones personales (ansiedad, sentimiento de culpa, rechazo personal, incapacidad vocacional) y dedicación preferente a otros enfermos con mejores perspectivas. También contribuyen factores institucionales como la relación coste-resultados, la planificación social, etc. De esta manera se produce un vacío asistencial en la atención a estos enfermos, originándose un grave problema para la medicina actual, no siempre bien resuelto. No es de extrañar que se hayan presentado diversas soluciones. Una de ellas ha sido la de reclamar la legalización de la eutanasia, que puede considerarse como la acción u omisión que, por su propia naturaleza o en sus intenciones, procura la muerte, con la finalidad de eliminar todo dolor y sufrimiento. Por definición la enfermedad terminal está irreversiblemente unida a la muerte; esto implica que médicos y enfermeros, además de administrar los cuidados físicos y psicológicos necesarios, han de esmerarse en evitar el sufrimiento y la inseguridad, así como acompañar hasta el final al enfermo agonizante... Actualmente se entiende por eutanasia aquella acción -eutanasia activau omisión -eutanasia pasiva- encaminada a dar muerte, de manera indolora, a los enfermos incurables. Son características esenciales de la eutanasia el ser provocada por personal sanitario y la existencia de una intencionalidad supuestamente compasiva o liberadora. Según la Organización Médica Colegial, "la eutanasia pasiva es verdadera eutanasia, pues, desde el punto de vista de la ética profesional, es irrelevante quitar la vida a un paciente mediante una acción que se ejecuta o mediante la omisión de una intervención médica obligada. En uno y otro caso hay eutanasia, pues se provoca deliberadamente la muerte de un paciente". La O.M.C. 
recomienda hablar de eutanasia (ya sea activa o pasiva) pues algunos "entienden, erróneamente, que eutanasia pasiva es no instaurar o suspender tratamientos médicos inútiles, y esto es un acto ético, por cuanto constituye práctica médica correcta"... Aunque sea con el consentimiento de la víctima, la eutanasia es siempre provocada por otras personas; es un homicidio con unas características determinadas. Si es uno mismo el que se provoca intencionadamente la muerte, se habla de suicidio y se denomina suicidio asistido cuando es el médico quien proporciona un fármaco letal al enfermo, pero es éste quien se lo administra a sí mismo. Como ya se ha mencionado, hoy en día, dentro del término "eutanasia" se incluyen conceptos de encontrada significación ética, y es necesario conocer el significado real del mismo, diferenciándolo de otras prácticas perfectamente admisibles desde un punto de vista ético y legal: No es eutanasia la aplicación de fármacos para aliviar el dolor u otros síntomas en un paciente terminal, aunque ello produzca, indirecta e inevitablemente, un cierto acortamiento de la vida. Si se aplican convenientemente los principios éticos, es no sólo aceptable sino aconsejable y necesario en ocasiones. Siempre debe procurarse no impedir que el enfermo pueda actuar libremente en la disposición de su última voluntad y, en el caso de que los medios usados lleven aneja la obnubilación o pérdida de consciencia, será necesario el consentimiento del enfermo. Tampoco es eutanasia la omisión o retirada de medios extraordinarios o desproporcionados para prolongar artificialmente la vida de un enfermo terminal, pues está ausente la acción positiva de matar y la posibilidad de una vida natural. A esto le llaman algunos autores "adistanasia". Es el médico -consultando en algunos casos límite a otros colegas-, o los comités de ética de algunos hospitales, los que deben determinar qué medios se pueden considerar proporcionados y cuáles desproporcionados para un paciente determinado, teniendo en cuenta sus circunstancias concretas. No obstante, hay una serie de medios que hoy día se consideran habitualmente como ordinarios o proporcionados (la hidratación y la nutrición -por boca o sonda nasogástrica- son los cuidados básicos mínimos). Más adelante se hablará con mayor amplitud de estos aspectos. Algunos llaman 
"ortotanasia" a la muerte a su tiempo, sin acortar la vida y sin alargarla innecesariamente mediante medios extraordinarios o desproporcionados. A este alargamiento de la vida mediante medios desproporcionados se lo conoce con el nombre de "ensañamiento terapéutico" o "distanasia" -lo contrario de la "adistanasia"-, y puede ser ilícito como señala el código deontológico. El "diagnóstico de muerte" es un punto importante ya que, desde que se establece el diagnóstico de muerte clínica, no hay problema de eutanasia, pudiéndose extraer los órganos del cadáver para transplantes (tras los requisitos legales pertinentes) o retirar la ventilación asistida. El cese irreversible de las funciones encefálicas y/o cardiorrespiratorias son criterios suficientes para confirmar la muerte de una persona, al cesar de funcionar su organismo espontáneamente como un todo. El tipo de enfermos en los que con mayor frecuencia se plantea la eutanasia son los que se hallan en situación terminal; conviene delimitar algunos conceptos al respecto. El término incurable hace referencia a la imposibilidad de mejorar o superar la enfermedad; terminal indica la cercanía de una muerte inevitable, aunque la enfermedad por su naturaleza pueda ser curable. Los enfermos incurables terminales son los principales candidatos a la eutanasia; los enfermos curables en estado crítico no presentan mayores dificultades, ya que habitualmente se les dan los cuidados máximos. Otro concepto es el de Estado Vegetativo Persistente (EVP); los pacientes pertenecientes a este grupo son incurables incapaces -el caso Schiavo- aunque no necesariamente son terminales, pues son pacientes con una pérdida de conciencia permanente, en coma irreversible. Un enfermo en situación terminal es aquel en que se prevé que la muerte es segura y ocurrirá en un plazo no lejano (hasta seis meses, según autores), abandonándose el esfuerzo médico terapéutico para concentrarse en el alivio de los síntomas y en el apoyo tanto al paciente como a su familia".

Como vemos, pocos son los autores que se decantan por defender o atacar la eutanasia de modo activo, ya que los límites entre el homicidio (justificable) y el acto humanitario son muy tenues. 
En la misma línea, los doctores López Martínez y Serrano Esteve del Hospital General de Valencia consideran en un trabajo de reciente publicación que "hasta hace poco el éxito o fracaso de las técnicas sanitarias se basaba casi exclusivamente en alargar la vida. Sólo muy recientemente se empieza a dar importancia a la calidad de vida. Hoy no se puede aceptar que el principio del respeto a la vida signifique el de su prolongación a cualquier precio. Cuando un enfermo manifiesta el deseo y la voluntad de morir, no es esto lo que primariamente busca, sino acabar con una serie de condicionantes que le hacen la vida demasiado dura e imposible. Es hacia estos condicionantes hacia donde debemos dirigir nuestros esfuerzos".

Añaden, más tarde, aspectos éticos al afirmar que "cuando se habla de problemas éticos $\mathrm{y}$, en particular, cuando se toca el tema de la eutanasia, se corre el riesgo de hacer interpretaciones a través de ideas religiosas o políticas, adoptando posturas intransigentes e incluso fanáticas. De esta forma, es difícil, hoy por hoy, enfocar el problema correctamente, ya que vivimos en una sociedad pluralista, donde conviven personas con distintas creencias religiosas y diferentes puntos de vista. Las modernas técnicas y los nuevos estilos de tratamiento hospitalario han deshumanizado la asistencia a los enfermos, colocándolos en una situación un tanto desfavorable a la hora de defender sus derechos. Cada vez más se habla del derecho de las personas a morir con dignidad que, desde el punto de vista ético, se refiere no al morir sino a la forma de morir, lo que implica una serie de principios:

a) Atención al moribundo con todos los medios posibles para aliviar el dolor y mejorar su situación clínica.

b) Facilitar que la muerte sea una acción personal.

c) Favorecer la vivencia del misterio humano-religioso de la muerte. 
d) No se puede privar al moribundo de la posibilidad de asumir su propia muerte.

Hoy no se puede aceptar que el principio del respeto a la vida, sobre el que está basada nuestra cultura, signifique el de su prolongación a cualquier precio".

Por lo tanto, no es de extrañar que concluyan afirmando que "el personal sanitario deberíamos respetar siempre que fuera posible, la voluntad del enfermo en lo referido al tratamiento que aplicar y los médicos deberían reflexionar seriamente antes de decidir sobre la prolongación de la vida o sobre la aceleración intencionada del proceso natural de la muerte"... "desde el momento en que se plantea la idea de que la vida humana, el nacimiento y la muerte son patrimonio divino, la eutanasia es tenida por delito, agravado o atenuado por las circunstancias que concurren en su práctica. Pero en el momento en que el hombre se siente dueño de su propio cuerpo y de su destino, y cuando las leyes le confieren la libertad para disponer de estos bienes, la eutanasia no se considera delito. Según esto, el consentimiento sería suficiente para anular el delito.

La polémica sobre la eutanasia tiene como base este enfrentamiento entre la religión, la moral, la ética y el libre pensamiento. La eutanasia es contraria a la doctrina de la Iglesia Católica desde el momento en que se tiene en cuenta el precepto "no matarás". Se condena el exterminio de los llamados "seres sin valor vital" aunque la omisión de esfuerzos para mantener artificialmente la vida (ortotanasia) sí que estaría aceptado. El derecho a la muerte humana no debe significar que se apliquen todos los medios disponibles, si el único fin de los mismos es el retrasar artificialmente la muerte. Mientras se pueda prever que con el uso de tales medios, ordinarios o extraordinarios, un enfermo se pueda curar, estaremos obligados a usarlos, pero en el caso de que no exista ninguna esperanza, no hay tal obligación, pues sólo 
retrasaríamos la muerte aumentando el sufrimiento. Podríamos preguntarnos al respecto: ¿El respeto a la vida humana exige provocar la terapia distanásica o, por el contrario, el derecho a morir dignamente postula la ortotanasia? Existen unas situaciones concretas en que la ortotanasia se presenta como mejor alternativa cuando mantener una vida puede llegar a convertirse en un acto inútil:

Vidas mantenidas mediante técnicas terapéuticas de reanimación: En estos casos si se llega a comprobar la muerte clínica del paciente, no tiene sentido mantener la vida puramente vegetativa. Incluso si no tiene lugar la muerte clínica del paciente, la reanimación sólo puede lograr en algunos casos una prolongación de una vitalidad parcial, reducida a reflejos casi exclusivamente vegetativos. En estos casos, la adistanasia no es moralmente reprobable $y$, a veces, es recomendable suspender el tratamiento adistanásico.

Situaciones en que el dejar morir es recomendable: Son casos en los que no hay obligación de prolongar la vida humana y se puede dejar morir al paciente, dependiendo de la esperanza que se tenga en la prolongación de la vida y de la cuantía del sufrimiento, etc.

El médico está obligado a proporcionar al enfermo los cuidados ordinarios para evitar su muerte y prolongar su vida: Esta obligación lo es también de la familia. Pero ni el médico ni la familia están obligados a recurrir a curas extraordinarias, en cuanto forman parte de tratamientos médicos altamente especializados.

Si el paciente está en coma prolongado e irreversible, cuya vida es totalmente vegetativa (caso Schiavo) y, aún en el caso de enfermos todavía conscientes que se encuentran en fase terminal de su enfermedad y que son mantenidos con vida artificialmente sin esperanza alguna de recuperación, no existe obligación de recurrir 
a medios extraordinarios o, si se ha recurrido a ellos, se pueden suspender legalmente.

La adistanasia supondría "dejar morir", mientras que la eutanasia se identificaría con "hacer morir".

Para concluir: "Con frecuencia los enfermos moribundos atraviesan diversas fases descritas por Kubler-Ross: negación, agresividad, pacto, depresión y aceptación. El enfermo puede expresar su deseo de morir en cualquiera de estas fases, pero la experiencia demuestra que muchos de aquellos que pedían la muerte en una fase de rebelión o desesperación, después de haberles sido aliviado el dolor físico y de sentirse acompañados, han encontrado significado a la última fase de su vida. Podríamos decir que la medicina paliativa correctamente aplicada sería el mejor antídoto de la eutanasia.

Antes de decidirse por practicar la eutanasia, deberían tenerse en cuenta un gran número de factores:

En primer lugar, y en caso de que el enfermo no se encuentre en condiciones óptimas para tomar la decisión por sí mismo, estaría la conciencia del médico y de los familiares o personas que deciden por el paciente. También habría que pensar si realmente la eutanasia se va a aplicar para evitar sufrimientos cuan do no existe esperanza alguna de curación o, si de manera encubierta, pudiese tomarse la decisión para librarse de una carga difícil de llevar por la familia.

Cuando ya se ha reflexionado suficientemente sobre el fin por el que se aplica la eutanasia, se debe valorar el grado de dolor y si el enfermo puede o no soportarlo. Por supuesto, cuando el enfermo está en condiciones de decidir sobre su futuro, hay que contar ante todo, con él. 
La adistanasia puede ser la mejor alternativa en pacientes en los que se ha comprobado la muerte clínica. Como ya hemos comentado, el médico está obligado a proporcionar al enfermo los cuidados ordinarios para evitar su muerte, prolongando su vida, pero no está obligado a recurrir a curas extraordinarias cuando ya no hay ninguna esperanza de recuperación.

El deber del personal sanitario y de la familia debe ir encaminado más a evitar sufrimientos y dolores evitables al enfermo que a prolongar su vida, con cualquier medio, el mayor tiempo posible una vez se haya constatado que esta vida se dirige inevitablemente hacia su extinción".

Bien otro es el caso de Ramón Sampedro, parapléjico gallego en cuyo caso de suicidio asistido (buscando la eutanasia) se basa la renombrada y oscarizada "Mar adentro" de Alejandro Amenábar (2004). Este enfermo, cuyo cuerpo paralizado de cuello hacia abajo le impedía cualquier movimiento, deseaba, en pleno uso de razón, una muerte rápida que le evitara años de estar postrado en la cama de manera irreversible (quizá los nuevos avances en la creación de tejido nervioso llevados a cabo en laboratorios estadounidenses pueden en un futuro lejano reconstruir la médula espinal dañada) tras un accidente en que se partió la columna vertebral.

¿Qué es más humano?, ¿complacer su deseo de no verse más como un vegetal con consciencia y no perjudicar a su familia y allegados, sin esperanza de recuperación?, ¿obligarle a vivir contra su voluntad, eliminando el bíblico concepto del libre albedrío?...

Las respuestas son tantas, a buen seguro, como lectores tenga este artículo. 
Esperemos que este estudio haya podido servir para aclarar alguno de los aspectos capitales a la hora de debatir qué es y qué no es eutanasia y ayudar a que se forme una opinión pública responsable, principio rector de toda democracia. 